\title{
Effect of Preparation Method on TEM Specimen
}

\author{
G. Wang, ${ }^{*}$ D.J. Llewellyn, ${ }^{* *}$ J.D. FitzGerald ${ }^{* * *}$ and Z.H. Stachurski**** \\ ${ }^{*}$ School of Materials Science and Engineering, Harbin Institute of Technology, Harbin, 150001, \\ China \\ ** Electron Microscopy Unit, Australian National University, Canberra ACT 0200, Australia \\ ${ }^{* * *}$ Research School of Earth Science, Australian National University, Canberra ACT 0200, \\ Australia \\ **** Department of Engineering, Australian National University, Canberra ACT 0200, Australia
}

We have investigated the amorphous phase of bulk metallic glass $\mathrm{Zr}_{41.25} \mathrm{Ti}_{13.75} \mathrm{Ni}_{10} \mathrm{Cu}_{12.5} \mathrm{Be}_{22.5}(\mathrm{BMG})$. However, this examined phase is metastable [1], its microstructural evolutions are easily activated due to the influence of applied stress [2], environmental heat [3], machining [4], ion milling [5] and so on. We have examined the influences of TEM specimen preparation techniques on the microstructural evolution of the BMG.

The Differential Scanning Calorimetry measurements were performed with a Perkin-Elmer Pyris 1 DSC. TEM observations were conducted on a Philips CM300 and a Philips EM430 machines both operating at $300 \mathrm{kV}$. One group of specimens were mechanical thinned and polished and then dimpled to a thickness of less than $15 \mathrm{um}$. This was followed by ion milling at an incident angle of $5^{0}$ for approximately $9 \mathrm{~h}$ to electron transparency at room temperature using a Gatan 691 Precision Ion Polishing System. Another group of specimens were prepared by dispersing finely ground BMG powders onto holey carbon film supported by a microgrid. The toughness of BMG material prevented crushing of solid pieces using a mortar and pestle, instead powder was produced by grinding on a new sheet of diamond lapping film. The abrasive particles contaminating the powder were easily distinguished (and therefore avoided) in the TEM based on their different appearance and chemical composition.

Representative TEM observations of the $\mathrm{Zr}_{41.25} \mathrm{Ti}_{13.75} \mathrm{Ni}_{10} \mathrm{Cu}_{12.5} \mathrm{Be}_{22.5} \mathrm{BMG}$ prepared by grinding are shown in Fig. 1a and b. At high magnification, the TEM image exhibits a maze-like pattern at the sub-nanomatre scale as shown in Fig. 1a. Fig. 1b shows a corresponding SAED pattern with two diffraction rings. All these features are consistent with an inherent amorphous character of the BMG. The ion milled BMG shows a quite different character (see Fig. 1c) - some patterns of thickness variation (presumed due to preferred etching) now dominate the TEM image. Also, the corresponding SAED pattern shows more weak diffraction rings appear at higher diffraction angles (see Fig. 1d), indicating early stages of the nanocrystallization [7].

To further characterize the structure of the ion milled BMG, DSC analysis was carried out to monitor the crystallization process. Fig. 2 shows the DSC traces of the BMG prepared by ion milling. The ion 
milled specimen for DSC measurement is carefully cut from the center part of the TEM specimen by a nail clipper because the outer rim of the TEM specimen was not exposed to the ion beam. In comparison, the DSC of the as-cast BMG is also shown in Fig. 2. It is obvious that the ion milled BMG exhibits the first exothermic peak disappearing, which indicates that the primary crystallization has occurred [7]. Although Li et al. have found that the ion milling could induce more structural order than electropolishing [5], it is observed that the ion milling can also induce the primary crystallization as observed in the present study. We speculate that localized heating of BMG specimens can occur even during low temperature ion milling [8].
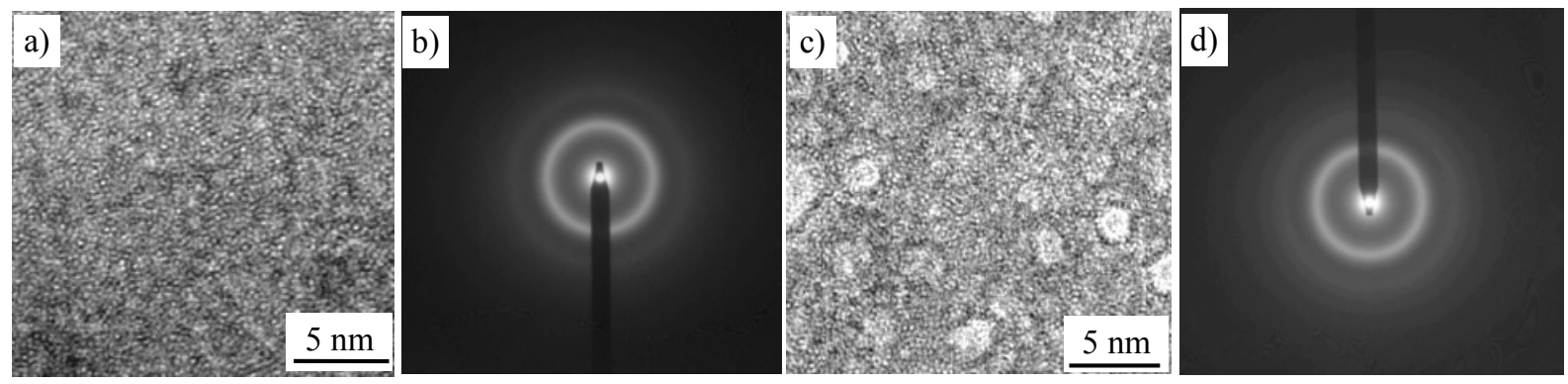

FIG. 1. TEM images of the BMG specimens prepared from various methods: a) and b) prepared by grinding; c) and d) prepared by ion thinning.

In order to find the reason for the thickness variations (see Fig. 1c), energy dispersive X-ray (EDX) spectrum analysis was used to check for chemical composition change. Ion milling has clearly implanted Argon onto the TEM specimen surface.

We also observed an electropolished TEM specimen. The TEM image and DSC trace are similar to those observed in ground-up specimen (not shown), which suggests that the electropolishing process does not affect the microstructure of the amorphous phase.

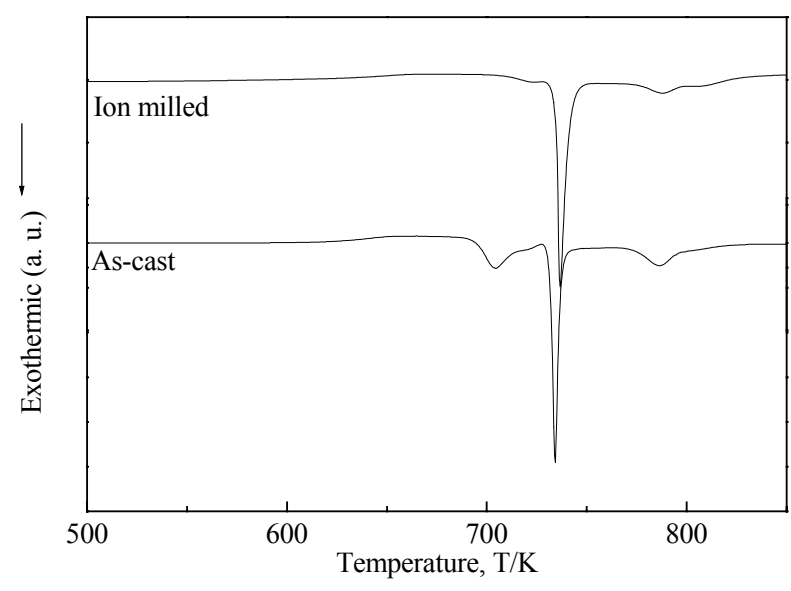

FIG. 2. DSC traces for the BMG specimens prepared from various methods.

For TEM observation of small regions in BMGs, the ground-up powder can be produced in just a few seconds and deposited on a carbon film. In addition, the grinding technique can avoid generating artifacts influencing the TEM observation. Although the electropolishing technique can avoid producing artifacts, its process is complicated as compared with the grinding technique. Therefore, for BMGs, the better TEM specimen preparation technique is dispersing ground BMG powder onto carbon film. 


\section{References}

[1] F.E. Luborsky, Amorphous metallic alloys, Butterworths, London, 1983.

[2] Y.F. Deng, L.L. He, Q.S. Zhang, H.F. Zhang, H.Q. Ye, Ultramicrosc. 98 (2004) 201.

[3] Y.L. Gao, J. Shen, J.F. Sun, D.M. Chen, G. Wang, et al., Mater. Lett. 57 (2003) 2341.

[4] M. Bakkal, A.J. Shih, R.O. Scattergood, C.T. Liu, Scr. Mater. 50 (2004) 583.

[5] J. Li, X. Gu, T.C. Hufnagel, Microsc. Microanal. 9 (2003) 509.

[6] A. Peker, W.L. Johnson, Appl. Phys. Lett. 63(17) (1993) 2342.

[7] I. Martin, T. Ohkubo, M. Ohnuma, B. Deconihout, K. Hono. Acta Mater. 52(15) (2004) 4427.

[8] D.J. Barber, Ultramicrosc. 52 (1993) 101.

[9] The assistance of staffs and use of TEM in the Electron Microscope Unit at ANU is acknowledged. 